\title{
Dynamic Alignment Using External Socket Reaction Moments in Trans-Tibial Amputees
}

\author{
Jonkergouw, N 1,2,3,4 ${ }^{*}$ Prins, M.R. 2,3,5 van der Wurff, P 2,5 Gijsbers, J ${ }^{6}$ Houdijk, H. ${ }^{3,} 7$ Buis, A.W.P. 4
}

1. Orthopedie Techniek Aardenburg, Military Rehabilitation Centre 'Aardenburg', Doorn, the Netherlands

2. Department of Research and Development, Military Rehabilitation Centre 'Aardenburg', Doorn, the Netherlands

3. Department of Human Movement Sciences, Faculty of Behavioral and Movement Sciences, Vrije Universiteit Amsterdam, Amsterdam Movement Sciences, the Netherlands

4. Department of Biomedical Engineering, Faculty of Biomechanical Engineering, University of Strathclyde, Glasgow, Scotland, United Kingdom.

5. Institute for Human Movement Studies, HU University of Applied Sciences Utrecht, the Netherlands

6. Motek Medical, Amsterdam, the Netherlands

7. Department of Research and Development, Heliomare, Wijk aan Zee, the Netherlands

Address all correspondence to Niels Jonkergouw; Department of Orthopedic Technology; Military Rehabilitation Centre 'Aardenburg'; Korte Molenweg 3, 3941 PW Doorn, the Netherlands; tel.: +31 343598 513; fax: 0031343598 438. Email: n.jonkergouw@mrcdoorn.nl

The views expressed in this article are those of the author(s) and do not necessarily reflect the official policy or position of the Ministry of Defense, Military Health Care Organization, or the Dutch Government.

\section{Highlights}

- Aligning trans-tibial prostheses by a predetermined kinetic criterion is eligible

- Induced alignment changes didn't affect spatiotemporal and kinematic gait parameters

- Prosthetic alignment changes had variable effects on knee moments and socket comfort

- The usability of External Socket Reaction Moments in the clinic is currently limited 


\section{Abstract}

Background: Prosthetic alignment is used to optimize prosthetic functioning and comfort. Spatio-temporal and kinematic gait parameters are generally observed to guide this process. However, they have been shown to be influenced by compensations, which reduces their sensitivity to changes in alignment. Alternatively, the use of moments working at the base of the prosthetic socket, external socket reaction moments (ESRM), has been proposed to quantify prosthetic alignment.

Research question: to investigate if a predetermined kinetic alignment criterion, $0 \mathrm{Nm}$ averaged over the stance phase, can be used to fine-tune prosthetic alignment.

Methods: 10 transtibial amputees were included in this intervention study. Firstly, their prostheses were aligned using conventional alignment procedures. Kinetic parameters and Socket Comfort Score (SCS) were measured in this initial alignment (IA) condition. Subsequently, the coronal plane ESRM during gait was presented to the prosthetist in real time using a Gait Real-time Analysis Interactive Lab. The prosthetist iteratively adapted the prosthetic alignment towards a predetermined average ESRM during the stance phase of $0 \mathrm{Nm}$. At the Final Alignment (FA), kinetic parameters and SCS were measured again and a paired sample t-test was performed to compare ESRMs and SCSs between alignments.

Results: A significant $(\mathrm{p}<0.001)$ change was found in the absolute coronal plane ESRM (mean \pm SD) from IA $(|0.104| \pm 0.058 \mathrm{Nm} / \mathrm{kg})$ to $\mathrm{FA}(|0.012| \pm 0.015 \mathrm{Nm} / \mathrm{kg})$. In addition a significant $(p<0.001)$ change of the external coronal adduction knee moments was observed from IA $(-0,127 \pm$ $0.079 \mathrm{Nm} / \mathrm{kg})$ to FA $(-0.055 \pm 0.089 \mathrm{Nm} / \mathrm{kg})$, however this change was more variable among participants. On average, no significant ( $p=0.37$ ) change in the SCS was observed.

Significance: While this study shows the potential of quantifying and guiding alignment with the assistance of kinetic criteria, it also suggests that a sole reliance on the ESRM as a single alignment criterion might be too simple.

Abbreviations: ESRM = External Socket Reaction Moment, FA = Final Alignment, GRAIL = Gait Real-Time Analysis Interactive Lab, IA = Initial Alignment, $\mathrm{Nm} / \mathrm{kg}=$ Newton meter per kilogram, SCS $=$ Socket Comfort Score

Key words: Alignment, Gait Analysis, Prosthetic, Quantification, Trans-tibial. 


\section{Introduction}

Trans-tibial prosthetic alignment, i.e., changes made to the position of the prosthetic foot relative to the socket, is performed with the aim of optimizing prosthetic comfort, stump-socket pressure distribution, energy expenditure, and gait stability. However, it is well established that reproducibility of the dynamic alignment process is limited [1,2]. This could be accredited to the iterative process of alignment based on the clinician's experience and tacit knowledge. Constructive efforts have been made to structure the process of alignment, which normally flows through three phases. During the first stage, "bench alignment," the prosthetic components are assembled and positioned in such a way that the user is able to stand with the prosthesis. This is followed by "static alignment" to determine whether the ground reaction force is orientated correctly relative to the different joints during an upright standing posture[3]. The final phase is the "dynamic alignment" which is used to optimize or fine tune, the prosthetic system during actual walking.

While bench alignment and static alignment can be well standardized $[4,5]$, variation in the final alignment after dynamic alignment remains high[1]. The dynamic alignment is currently executed by using the subjective interpretation of various kinematic and spatio-temporal parameters (STP) during gait. Subsequently, alignment is adjusted if asymmetries and/or deviations of the kinematics and STP relative to normal walking are observed. However, amputees show some adaptability and preserve consistent spatiotemporal and kinematic gait parameters irrespective of the induced alignment change [6-8]. The use of these outcomes to optimize the alignment is therefore debatable[2]. Although adaptations in the gait pattern can obscure effects of alignment on kinematic parameters, these effects remain observable in the underlying kinetic source data.

Therefore, it might be important to investigate the possible use of kinetic parameters to align lower limb prostheses and optimize prosthetic gait. Kobayashi et al [9] introduced External Socket Reaction Moments (ESRMs) as a way to quantify prosthetic alignment. These ESRMs are measured at the tube-socket connection and vary in sagittal, coronal, and transversal planes throughout the gait cycle. It has been reported that small alignment changes had a significant influence on these moments. Therefore it was argued that they could be useful to quantify alignment changes induced within a trans-tibial prosthesis during dynamic alignment $[7,9,10]$. 
Multiple studies have confirmed the sensitivity of these ESRMs to systematically induced alignment changes [9-12]. They all report predictability of alignment changes on ESRMs during gait and hint towards using ESRMs as a measurement to quantify prosthetic alignment. However, it remains unknown what the optimal mean value or pattern of the ESRM for a specific amputee should be [11]. Moreover, to date, studies have only investigated which changes in ESRM occur as a consequence of given alignment changes, but not the reverse, that is, whether a given ESRM can be reached by a series of subsequent alignment changes. Therefore, it is interesting to investigate whether it is possible to use a predefined ESRM optimization criterion for multiple trans-tibial prosthetic users.

The goal of this study was to investigate whether it was possible to minimize the coronal mean ESRM, towards a $0 \mathrm{Nm}$ average over the stance phase, by systematic alignment changes. The predetermined kinetic alignment criterion of $0 \mathrm{Nm}$ is a relatively arbitrary guess, which anecdotally has been related to minimizing stump socket reaction forces during gait. Although the main objective of this study was to test whether predetermined criteria can be reached through a series of alignment changes performed by a prosthetist, we additionally assessed the effect of the selected criterion on socket comfort score (SCS)[13]. While the alignment criterion focused on minimizing the moments at the base of the socket, this might also have a considerable influence on the orientation of the ground reaction vector on more proximal joints. Therefore, to explain possible changes of the SCS, the knee moments in the coronal plane were monitored as well.

\section{Methods}

\section{Subject inclusion criteria}

Participants were recruited by a call for participation at the forum of the Dutch Association of Amputees. Participants had a unilateral trans-tibial amputation and had been using their prosthesis for at least 1 year. They were not suffering from stump problems and were able to walk without additional assistive devices. Potential participants used a modular endo-skeletal prosthesis with a pin-fixation in addition to an Elastic Response Foot. All participants were over 18 years of age and able to provide informed consent.

Ethical approval was obtained from the Medical Ethical Testing Committee Brabant, Tilburg, the Netherlands (NL50704.028.14, P1451) along with approval from the University Ethical 
Committee, University of Strathclyde, Scotland (UEC15/14). Informed consent was obtained from all participants.

\section{Data acquisition}

\section{Gait Real-time Analysis Interactive Lab (GRAIL)}

Data acquisition was performed on the Gait Real-Time Analysis Interactive Lab (GRAIL) (Motek Forcelink, Amsterdam, the Netherlands). This system consists of an instrumented dualbelt treadmill, with 10 high-frequency infrared motion capture cameras (Vicon, Oxford Metrix, United Kingdom) and a $240^{\circ}$ projection screen. A safety harness system was suspended above the treadmill. Although the harness was attached, no additional support was provided. Data were collected at $100 \mathrm{~Hz}$.

\section{Marker models}

We used two marker models: (1) the Human Body Model [14], to capture STP, kinematic, and kinetic parameters of the lower extremities during initial (IA) and final alignment (FA), and (2) the ESRM model, to capture the ESRM during gait. We required 25 reflective markers and data from two forceplates for the Human Body Model. These markers were labeled and tracked using Nexus (VICON). Subsequently, joint kinematics and kinetics were calculated in the D-Flow software 3.26 (Motek Forcelink).

The ESRM model required six markers and calculated the moments at the base of a socket, which is not a known joint center within a standardized model. Therefore, an additional model was developed to calculate these ESRMs during alignment (Appendix I). To calculate the mean ESRM moment in real-time the GRAIL was controlled by D-Flow software, which integrated the data from force plates and motion-capture systems.

\section{Protocol}

\section{Instrumented prosthesis}

An experimental prosthesis was manufactured, in which the prosthetic socket and pin system of the participant's prosthesis were reused. All prosthetic elements directly below the anchor of 
the pin system were replaced as follows (Figure 1): (1) an iPECs lab $(450 \mathrm{~g})^{\dagger}$, connected to the pyramid of the prosthetic (2) pin system by screws in the coronal plane and (3) customized $70 \mathrm{~mm}$ tubes with reflective markers in the sagittal plane to facilitate inverse dynamic calculations. An OB4R1 (4) alignment jig (600g) was attached to allow coronal plane translation adjustments (+/$25 \mathrm{~mm}$ ) and coronal angulation $\left(+/-6^{\circ}\right)$. A pylon, its length dependent on the participant's height and stump length (5), was used in between the jig and a (6) triton low profile foot. All participants were provided with the same (7) shoes (Dachstein).

Insert figure 1: Instrumented prosthesis

\section{Walking speed determination}

The experiment started with a warm-up trial of approximately 5 minutes of walking on the GRAIL. The comfortable walking speed of the participant was determined with the participant's daily life prosthesis and shoes. The initial treadmill speed was set to $1.0 \mathrm{~km} / \mathrm{h}$, which was then gradually increased until the participant indicated that a comfortable walking speed had been reached. Subsequently, this speed was increased by $1.5 \mathrm{~km} / \mathrm{h}$ and slowly decreased until a comfortable speed was reached. The mean of these two subjective values determined the comfortable walking speeds for that participant [15].

\section{Protocol}

One experienced prosthetist $(\mathrm{XX})$ received all prosthetic components and performed a bench, static, and dynamic prosthetic alignment, without the use of the instrumented prosthesis until the prosthetist and participant were satisfied with the (IA). A gait analysis on the GRAIL followed, during which kinematics and kinetics of the lower limbs as well as the ESRM of the prosthesis were obtained at the participant's comfortable walking speed. A SCS [13] was administered after the trial. To quantify the position of the foot in relation to the socket, its orientation was captured by the GRAIL system after IA and FA.

The IA gait analysis was superseded by the computer-assisted alignment approach during which the prosthetic alignment was tuned towards the mean external socket reaction of $0 \mathrm{Nm}$ during the prosthetic stance phase (Appendix I). The D-flow application calculated the mean ESRM of the IA and showed the ESRM in real time on the GRAIL's screen (out of sight of the participant).

\footnotetext{
† The iPecs system was used for validation of our inverse dynamics ESRM model and is discussed in appendix I
} 
This allowed the prosthetist to determine the necessary alignment adjustments reflected by a change of ESRM.

Angulations change the rotation as well as translation of the foot relative to the socket. Therefore, adjustments were separated in angulations and translations. With a mean ESRM $>5$ $\mathrm{Nm} / \mathrm{kg}$, coronal angulations were performed, while medio-lateral translations were used with a mean ESRM $<5 \mathrm{Nm} / \mathrm{kg}$. Adjustments were executed until two consecutive changes failed to bring the mean ESRM closer to $0 \mathrm{Nm}$.

The resulting alignment was defined as the FA. A gait analysis in this FA condition was performed, the foot orientation was measured, and the SCS was administered. If a change of SCS was detected compared to IA the participant was asked to give an elaboration of what was experienced.

\section{Statistical analysis and data analysis}

The Kolmogorov-Smirnov normality test and a visual inspection of the normal Q-Q plots were used to test the distribution of age, body weight/height, and stump and foot length, and a normal group distribution was found. Therefore, a paired sample $t$-test was performed to test the differences in SCS, absolute mean ESRM and external knee moment comparing IA versus FA.

The ESRM and knee-moment data were investigated in the local coronal plane and were normalized to body weight. Statistical analyses were performed using SPSS 21.0 (SPSS Inc., Chicago, IL). Statistical significance was set to an alpha of 0.05 for all statistical tests.

\section{Results:}

\section{Participants:}

Ten participants with unilateral trans-tibial amputation were recruited (Table 1). Seven had an amputation due to trauma, two because of peripheral vascular disease and one as a result of a congenital defect. All participants were classified with moderate (K3) to high (K4) activity levels and they all used an Elastic Response Foot.

Insert Table 1: Group Demographics

\section{Spatiotemporal gait data}


The average comfortable walking speed was $4.12( \pm 0.56) \mathrm{km} / \mathrm{h}$. For both conditions (IA and FA) the prosthetic limb stance phase was significantly longer compared to the non-prosthetic limb. There was no significant change of the spatiotemporal gait parameters from IA to FA (Table 2).

Insert Table 2: Spatiotemporal gait parameters in both conditions and normalized ESRM values in IA and FA conditions

\section{External Socket Reaction Moment change}

A significant $(\mathrm{p}<0.001)$ reduction of the average absolute ESRM from IA $(|0.104| \pm 0.058 \mathrm{Nm} / \mathrm{kg})$ to FA $(|0.012| \pm 0.016 \mathrm{Nm} / \mathrm{kg})$ was found. Moreover, a reduction of the standard deviation is also shown (Table 3, Figure 2).

Insert Figure 2: Mean External Socket Reaction Moment (A), Socket Comfort Score (B) and mean Knee Moment ( $C$ )

Insert Figure 3: Mean ESRM and knee moments averaged over all participants across stance $(0-100 \%)$

\section{Mean external coronal knee moments}

A significant effect of the alignment process was found for external coronal net joint knee moments (Figure 2). On average, the group mean adduction-oriented coronal knee external moments significantly $(\mathrm{p}<0.001)$ decreased in magnitude from IA $(0.127 \pm 0.079 \mathrm{Nm} / \mathrm{kg})$ to $\mathrm{FA}$ $(0.055 \pm 0.089 \mathrm{Nm} / \mathrm{kg})$.

Participant 2 experienced a large directional change of the mean knee moment from adduction in IA $(0.019 \mathrm{Nm} / \mathrm{kg})$ to a mean abduction moment in the FA condition $(-0.096 \mathrm{Nm} / \mathrm{kg})$ (Figure 3). Two other participants (5 and 6) also experienced a directional change of the mean external knee moment from adduction towards abduction in the FA condition as well. However, the magnitude of these moments was close to zero. All other participants experienced a decrease of the mean external knee adduction moment in the FA.

\section{Alignment changes}


The mean change $( \pm S D)$ of the foot relative to the socket was $1.32( \pm 0.90) \mathrm{cm}$ lateral translation and $0.82 \pm 1.25^{\circ}$ angulation at the tube socket connection, moving the foot and tube towards adduction and laterally relative to the prosthetic socket.

\section{Socket comfort score}

There was no significant change $(p=0.37)$ in the SCS (mean \pm SD) from the IA $(7.6 \pm 1.0)$ to FA

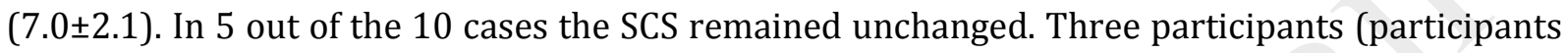
2,7 , and 10) reported a worsening of the SCS at the FA (Figure 2). Participant 2 reported a decrease of five points, related to an uncomfortable and unstable walking pattern. Participants 7 and 10 explained their decrease in comfort by the necessity of an additional residual limb sock, due to a reduction in stump volume. Participants 1 and 3 reported an improvement of the SCS, explained by a subjectively better internal socket force distribution.

\section{Discussion}

This study demonstrates the feasibility of alignment to a predetermined kinetic alignment criterion, the ESRM, for unilateral trans-tibial prosthetic users. We selected an arbitrary criterion of $0 \mathrm{NM}$ and succeeded to reduce the ESRM significantly towards zero for all participants. This was reached through substantial manipulation of the alignment. Although we do not claim that this criterion would be an optimal target for alignment nor do we aim to investigate optimal alignment in this study, interesting observations were made on the usability of this kinetic alignment criterion in practice. These observations will be discussed below.

The direction of our ESRM's (varus) in Initial Alignment is in agreement with previous published articles $[10,16]$. IA in this study shows that it is possible to reduce the ESRM substantially and significantly towards $0 \mathrm{Nm}$ for all included participants. However, substantial alignment changes from IA were necessary to achieve this research outcome. Despite that, no significant changes in the spatiotemporal gait pattern were reported. These findings support the conclusions drawn in a recent systematic review [2], highlighting the adaptability of the human body. Prosthetic users conceal the effect of alignment changes and are shown to regulate and preserve their kinematics and self-selected step characteristics despite the large changes in the alignment of the prosthesis. 
In contrast, the induced alignment changes did have a considerable influence on the orientation of the ground-reaction force vector relative to the joints proximal to the socket-tube interface. The criterion of mean ESRM close to zero requires the ground-reaction force vector to run closely to the tube-socket connection, which was indeed observed in this study. However, simultaneously the alignment changes had a substantial influence on the external knee adduction moment, which on average decreased significantly over all participants, however large variations in knee moment occurred between amputees.

Evidently, fine-tuning alignment relative to the socket-tube connection (i.e. the anchor) results in a low variation between amputees on ESRM data in FA. However, the position of the anchor relative to the stump and knee joint can vary considerably between patients, e.g. anatomical differences between amputees and the arbitrarily placement of the anchor. As such variation in anchor positioning will result in different effect of a 0Nm ESRM on the moment around the proximal joints (figure 4). Given this large standard deviation observed in the knee joint moment at FA, it can be concluded that a kinetic parameter measured distally to the socket (i.e., ESRM) is not a direct predictor of kinetics on more proximally orientat2ed joints. It is in our opinion therefore, impossible to suggest alignment changes by ESRM as a singular alignment criterion. This would also suggest individualization of prosthetic alignment is necessary if ESRMs are used. Which is in agreement with Kobayashi et al (2014) who suggested that individuals responded differently to alignment perturbations, with a different foot-socket orientation at FA [11].

To clarify this, we highlighted one of the participants (\#2) who experienced a large (five points) decrease in the SCS. For this individual, the decrease of the ESRM around the base of the socket induced a change of direction of the ground reaction force relative to the center of the knee joint, creating a mean external knee abduction moment instead of an adduction moment (Figure 2 and Appendix II). Because our alignment criteria aimed to fine-tune the ESRM working at the base of the socket, changes like this can occur depending on the position of the anchor on the distal end of the socket. As shown by this individual, a given specific ESRM criterion is unlikely to result in an optimal alignment for each individual.

Insert Figure 4: Theory of effect of ground reaction force on knee joint when achieving ONm with different anchor-socket orientations

The induced alignment changes (and concomitant changes in ESRM and knee moment) had no significant influence on the SCS for the majority of the participants, suggesting that there is no 
immediate effect on perceived socket fit during gait. This could imply that the SCS does not depend on these kinetic changes in and around the prosthetic socket and proximal joints. Nevertheless, stump problems could be present later on and effects on the SCS might have remained unnoticed in the short-term follow up of this study. It could therefore be argued that the SCS itself is not sensitive enough to investigate the immediate effect of externally induced changes on internal force distribution. Therefore, we suggest future alignment studies do not solely rely on the SCS and recommend measuring the effect of the induced changes on gait stability, energy expenditure, and kinetic forces on higher orientated joints in addition to forces within the socket/prosthetic system.

An important limitation is the lack of control of socket fit. During the measurements one patient experienced a drop of three on the SCS and explained this by the necessity to wear an additional stump-sock. In addition, due to the design of the study (FA occurs after IA) randomization was impossible. Therefore, stump-volume fluctuations could have some influence on this study. An additional limitation is that we were required to choose an arbitrary cut-off value between angulation and translation. To our knowledge there are no standardization guidelines available for this kind of research.

\section{Conclusion}

It was possible to fine-tune the prosthetic alignment of all participants towards $0 \mathrm{Nm}$. However, minimizing the ESRMs did not have a significant effect on the SCS. The concomitant change in foot orientation had a varying effect on the moments acting around more proximally orientated joints. While this study shows the potential of quantifying alignment with the assistance of kinetic criteria, it also suggests that a sole reliance on the ESRM as a single alignment criterion might be too simple. The limitation of using ESRM criteria was discussed, and we showed that additional parameters, such as the kinetics of more proximal joints, should be taken into account during computer-aided dynamic alignment.

\section{Conflict of Interest statement}

The authors have declared that no competing interests exist.

Declarations of interest: none

Funding: This research did not receive any specific grant from funding agencies in the public, commercial, or not-for-profit sectors. 


\section{Contributions:}

N. Jonkergouw: Concept and design of the study, analysis and interpretation of data, drafting/revising of manuscript and final approval

M.R. Prins

Concept and design of the study, analysis and interpretation of data, revising of manuscript and final approval

P. van der Wurff:

Concept and design of the study, revising of manuscript and final approval

J. Gijsbers

Concept and design of the study, revising of manuscript and final approval

H. Houdijk:

Concept and design of the study, revising of manuscript and final approval

A.W.P. Buis:

Concept and design of the study, revising of manuscript and final approval

Declarations of interest: none

Funding: This research did not receive any specific grant from funding agencies in the public, commercial, or not-for-profit sectors.

Acknowledgements: The authors would like to acknowledge the assistance of Lotte Jonkergouw, Tommie Perenboom for their assistance in formatting the figures and Bente $\mathrm{v} / \mathrm{d}$ Wurff for editing the language used within the article. 


\section{Literature}

[1] M. Zahedi, W. Spence, S. Solomonidis, J. Paul, Alignment of lower-limb prostheses, J. Rehabil. Res. 23 (1986) 2-19.

[2] N. Jonkergouw, M.R. Prins, A.W.P. Buis, P. Van Der Wurff, The Effect of Alignment Changes on Unilateral Transtibial Amputee's Gait: A Systematic Review, PLoS One. 11 (2016). doi:10.1371/journal.pone.0167466.

[3] S. Blumentritt, A new biomechanical method for determination of static prosthetic alignment, Prosthet. Orthot. Int. 21 (1997) 107-113.

[4] S. Blumentritt, T. Schmalz, R. Jarasch, M. Schneider, Effects of sagittal plane prosthetic alignment on standing trans-tibial amputee knee loads, Prosthet. Orthot. Int. 23 (1999) 231-238.

[5] S. Blumentritt, T. Schmalz, R. Jarasch, Influence of static prosthetic alignment on standing posture and walking in transtibial amputees, Orthopade. 30 (2001) 161-168. doi:10.1007/s001320050590.

[6] C. Grumillier, N. Martinet, J. Paysant, J.M. André, C. Beyaert, Compensatory mechanism involving the hip joint of the intact limb during gait in unilateral trans-tibial amputees, J. Biomech. 41 (2008) 2926-2931. doi:10.1016/j.jbiomech.2008.07.018.

[7] D.J. Sanderson, P.E. Martin, Lower extremity kinematic and kinetic adaptations in unilateral below-knee amputees during walking, Gait Posture. 6 (1997) 126-136. doi:10.1016/S0966-6362(97)01112-0.

[8] C. Beyaert, C. Grumillier, N. Martinet, J. Paysant, J.M. André, Compensatory mechanism involving the knee joint of the intact limb during gait in unilateral below-knee amputees., Gait Posture. 28 (2008) 278-84. doi:10.1016/j.gaitpost.2007.12.073.

[9] T. Kobayashi, M.S. Orendurff, M. Zhang, D.A. Boone, Effect of alignment changes on sagittal and coronal socket reaction moment interactions in transtibial prostheses., J. Biomech. 46 (2013) 1343-50. doi:10.1016/j.jbiomech.2013.01.026.

[10] D.A. Boone, T. Kobayashi, T.G. Chou, A.K. Arabian, K.L. Coleman, M.S. Orendurff, M. Zhang, Influence of malalignment on socket reaction moments during gait in amputees with transtibial prostheses, Gait Posture. 37 (2013) 620-626. doi:10.1016/j.gaitpost.2012.10.002.

[11] T. Kobayashi, M.S. Orendurff, M. Zhang, D. a Boone, Individual responses to alignment perturbations in socket reaction moments while walking in transtibial prostheses., Clin. 
Biomech. 29 (2014) 590-4. doi:10.1016/j.clinbiomech.2014.04.002.

[12] T. Kobayashi, M.S. Orendurff, D.A. Boone, Dynamic alignment of transtibial prostheses through visualization of socket reaction moments., Prosthet. Orthot. Int. Online (2014) 1-5. doi:10.1177/0309364614545421.

[13] R.S. Hanspal, K. Fisher, R. Nieveen, Prosthetic socket fit comfort score, Disabil. Rehabil. 25 (2003) 1278-80.

[14] A.J. Van Den Bogert, T. Geijtenbeek, @bullet Oshri, E.-Z. @bullet, F. Steenbrink, E.C. Hardin, A real-time system for biomechanical analysis of human movement and muscle function, (n.d.).

https://www.ncbi.nlm.nih.gov/pmc/articles/PMC3751375/pdf/11517_2013_Article_107 6.pdf (accessed May 14, 2018).

[15] L. Hak, H. Houdijk, P. Van Der Wurff, M.R. Prins, P.J. Beek, J.H. Van Dieën, Stride frequency and length adjustment in post-stroke individuals : influence on the margins of stability, J. Rehabil. Med. 47 (2015) 126-132. doi:10.2340/16501977-1903.

[16] T. Kobayashi, A.K. Arabian, M.S. Orendurff, T.G. Rosenbaum-Chou, D. a Boone, Effect of alignment changes on socket reaction moments while walking in transtibial prostheses with energy storage and return feet., Clin. Biomech. 29 (2014) 47-56. doi:10.1016/j.clinbiomech.2013.11.005. 


\section{Table 1}

\section{Table 1: Group Demographics}

Group's mean value \pm standard deviation

$\begin{array}{llllllllll}\text { Participants } & \text { Gender } & \text { Age (y) } & \begin{array}{l}\text { Weight } \\ \text { (kg) }\end{array} & \begin{array}{l}\text { Height } \\ \text { (cm) }\end{array} & \text { Foot length (cm) } & \begin{array}{l}\text { Stump } \\ \text { length } \\ \text { (cm) }{ }^{1}\end{array} & \begin{array}{l}\text { Side } \\ \text { amputation }\end{array} \text { of } \begin{array}{l}\text { Cause } \\ \text { amputation }\end{array} \text { Activity } \\ \text { level }\end{array}$

Abbreviations: $\mathrm{cm}=$ centimeter,$F=$ Female, $\mathrm{kg}=$ kilograms, $\mathrm{km} / \mathrm{h}=$ kilometers $/$ hour,$M=$ Male, mo = months, SD = Standard Deviation, $y=$ years

${ }^{1}$ Stump length is determined by taking the length from the proximal tibia plateau to the distal tibia end. 


\section{Table 2}

Table 2: Spatiotemporal gait parameters in both conditions and normalized ESRM values in IA and FA conditions

All values are reported as a mean \pm standard deviation, for spatiotemporal parameter this is followed by the percentage of the gait cycle in parentheses. The step width accounts for both legs.

The ESRM change from initial alignment to final alignment and is reported for normal (mean and $\mathrm{p}$ ) and absolute values (|mean| and $|\mathrm{p}|$ ). All means are reported in $\mathrm{Nm} / \mathrm{kg}$ followed by a standard deviation. Negative is defined as an adduction oriented ESRM, where positive defines an abduction-orientated moment.

\begin{tabular}{|c|c|c|c|c|}
\hline & \multicolumn{2}{|c|}{ Prosthetic Limb } & \multicolumn{2}{|c|}{ Non-Prosthetic Limb } \\
\hline & IA-condition & FA-condition & IA-condition & FA-condition \\
\hline $\begin{array}{l}\text { Stance Duration } \\
\text { (seconds) }\end{array}$ & $0.73 \pm 0.10(65 \%)^{*}$ & $0.73 \pm 0.10(65 \%) *$ & $0.76 \pm 0.10(68 \%)^{+}$ & $0.76 \pm 0.09(68 \%)^{+}$ \\
\hline $\begin{array}{l}\text { Swing Duration } \\
\text { (seconds) }\end{array}$ & $0.39 \pm 0.02(35 \%) *$ & $0.40 \pm 0.03(35 \%) *$ & $0.36 \pm 0.02(32 \%)^{+}$ & $0.36 \pm 0.03(32 \%)^{+}$ \\
\hline $\begin{array}{l}\text { Step } \\
\text { (meters) }\end{array}$ & $0.10 \pm 0.02$ & $0.11 \pm 0.01$ & $\mathrm{~N} / \mathrm{A}$ & N/A \\
\hline Mean ESRM & $-0.102 \pm 0.061$ & $-0.011 \pm 0.017^{* *}$ & $\mathrm{~N} / \mathrm{A}$ & N/A \\
\hline $\begin{array}{l}\text { Absolute ESRM } \\
\text { change |mean| }\end{array}$ & $0.104 \pm 0.058$ & $0.012 \pm 0.016^{* *}$ & $\mathrm{~N} / \mathrm{A}$ & N/A \\
\hline
\end{tabular}

Abbreviations: $E S R M=$ External Socket Reaction Moment, FA=Final Alignment, IA=Initial Alignment, N/A = Not Applicable, Nm $/ \mathrm{kg}=$ Newton meter per kilogram.

${ }^{* *} \mathrm{p}<0.001$ from IA to FA for ESRM changes, ${ }^{*} \mathrm{p}<0.05$ vs Non-Prosthetic Limbs, ${ }^{+} \mathrm{p}<0.05$ vs Prosthetic Limbs, in the same condition 


\section{Figures}

Color print: Figure 1: Instrumented prosthesis 
Black/white print Figure 2: Mean External Socket Reaction Moment (A), Socket Comfort Score (B) and mean Knee Moment (C). All moments are calculated as the mean moment during stance phase and were normalized by body weight $(\mathrm{Nm} / \mathrm{kg})$. Note that the trend of ESRM and Knee Moment change is in the same direction for all participants. Participant 2 is particularly interesting and is highlighted since this participant will be discussed throughout the article 
Black/white print Figure 3: Mean ESRM and knee moments (positive is abduction) averaged over all participants across stance (0-100\%). This figure showcases the change from initial to final alignment. 


\section{Color print Figure 4: Theory of effect of ground reaction force on knee joint when achieving $0 \mathrm{Nm}$ with}

different anchor-socket orientations. Theoretically all above cases represent an ESRM of 0Nm. It showcases that achieving a predetermined $0 \mathrm{Nm}$ could have varying consequences on the moments working around higher orientated joints. Considering the effect of anchor placement as well as how the final alignment is achieved we detected this in our research data as well and highlighted 2 cases in our supplementary data (Appendix II)
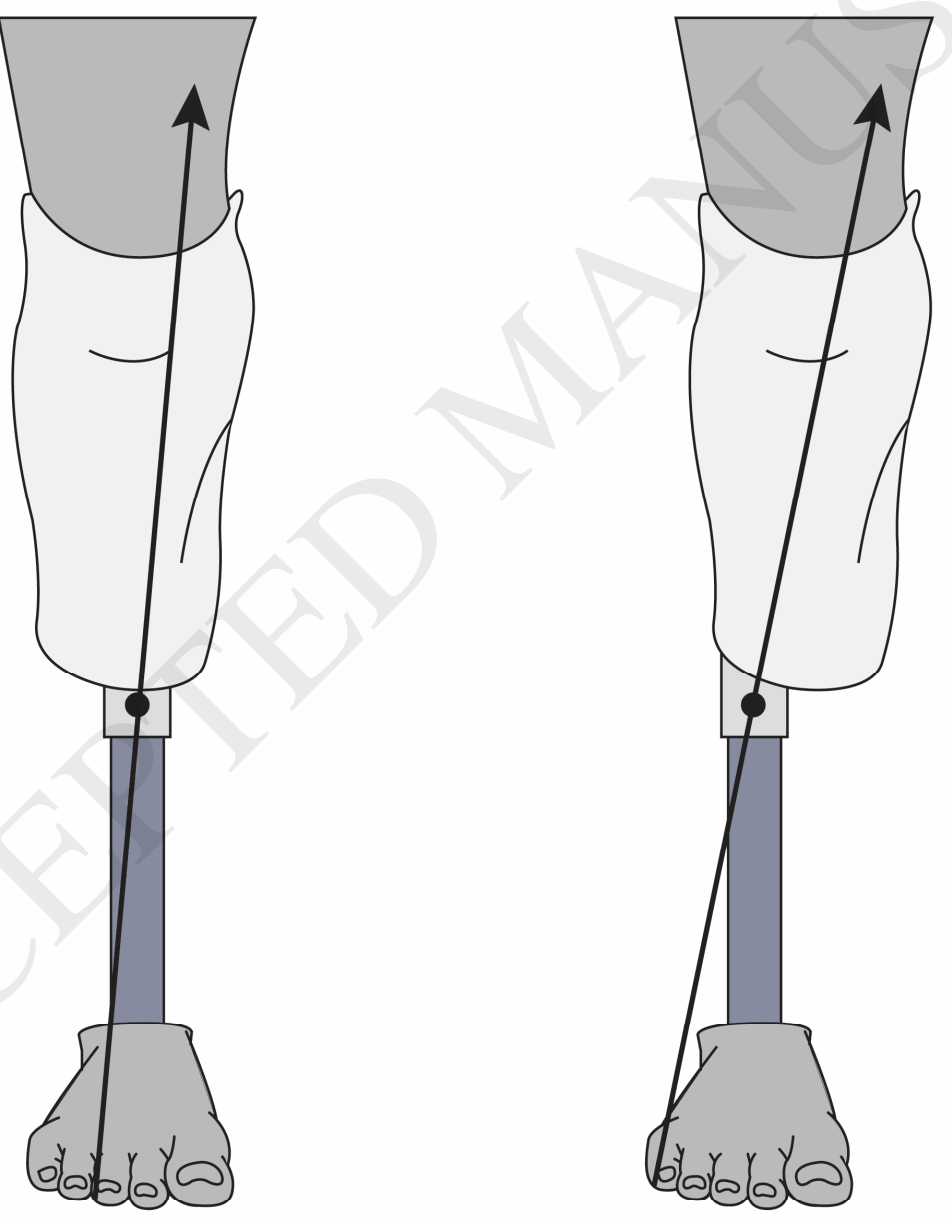

Figure 4: Theoretical effect of different anchor locations on external knee moment for a given ESRM Theoretically all above cases represent an ESRM of $0 \mathrm{Nm}$. It showcases that achieving a predetermined $0 \mathrm{Nm}$ could have varying consequences on the moments working around higher orientated joints. Considering the effect of anchor placement as well as how the final alignment is achieved. We detected this in our research data as well and highlighted 2 cases in our supplementary data (Appendix II) 


\section{Appendix I: ESRM-model}

A mathematical model of a below knee prosthetic limb was constructed to calculate the coronal mean ESRM during stance phase of the prosthetic limb (Figure 5), the ESRM-model. A total of six markers were placed on the prosthetic limb (Figure 1.1). Two of those markers are placed on a customized tube (Figure 1.2) and created a pre-set distance from the center of the socket tube transition. One marker is placed on each malleolus, one on the calcaneus and one on the second metatarsal bone. The positions of these markers were used to calculate the position of the pyramid's center. The pyramid center was used in combination with the force plates, measuring the ground-reaction vector, allowing calculation of the mean ESRM in the coronal plane. In order to test the validity of the ESRM model a pilot study was completed for one participant in preparation for this research. The mathematically derived data were comparable to the iPecs data and allowed our investigation to proceed with a larger group $(n=10)$
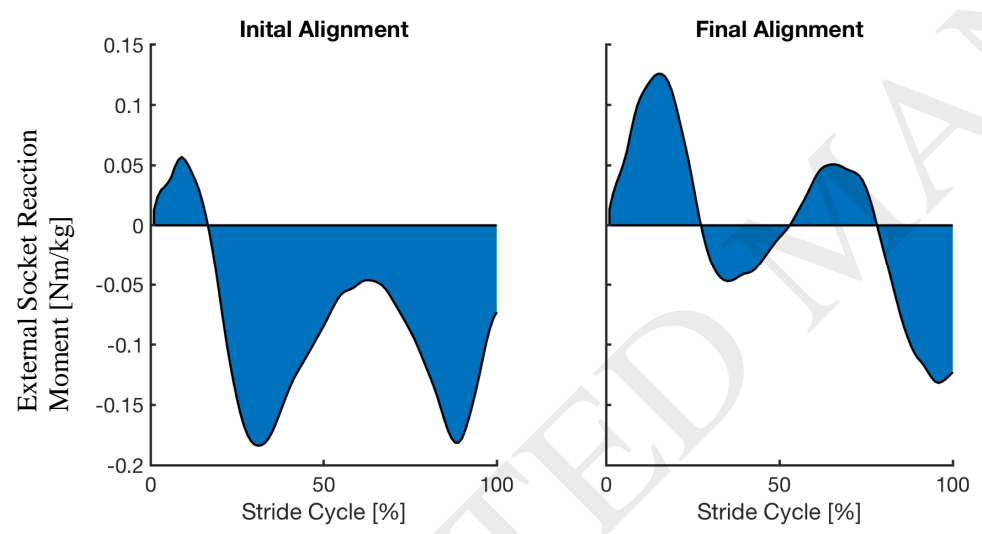

Figure 5: Example (Participant 1) of mean ESRM calculation during stance phase of prosthetic limb. The mean ESRM was calculated by taking the integral (area under the curve) per step in ( $\mathrm{Nm}^{*}$ s), dividing by stride time in (s); therefore the mean ESRM unit was (Nm). Heel strikes were identified as local peaks in the anteroposterior position of the calcaneus marker and toe-offs as local valleys of the anteroposterior position of the MTII marker.

Negative is defined as an adduction moment, where positive defines an abduction-oriented moment.

\section{Results of IPecs data for this study:}

The iPecs (Intelligent Prosthetic Endo-Skeletal Component System) is a six-axis load cell that accurately measures three-dimensional forces and moments experienced by a prosthesis user. The cell was integrated into the prosthesis, below the socket and above the foot, measuring the torques at the base of the socket as the user walks, the ESRMs (Figure 1.3). The cell has a similar functionality to the force plates used in a gait lab, except that it does not require an advanced gait 
laboratory (RTC-Electronics, 2014). The additional ESRM measured by the iPecs-system was used in this study for comparison with the ESRM-model.

\section{ESRM ipecs vs inverse dynamics}

For the iPecs method there was a significant $(\mathrm{p}=0.014)$ difference in the absolute ESRM from IA $(|0.077| \pm 0.041 \mathrm{Nm} / \mathrm{kg})$ to $\mathrm{FA}(|0.029| \pm 0.019 \mathrm{Nm} / \mathrm{kg})$. With similar results when using the inverse dynamics method, showing a significant $(p<0.001)$ difference of the absolute ESRM from IA $(|0.104| \pm 0.058 \mathrm{Nm} / \mathrm{kg})$ to FA $(|0.012| \pm 0.016 \mathrm{Nm} / \mathrm{kg})$. These results suggest that in both methods, the varus-orientated ESRM was significantly decreased towards the hypothesized moment of $0 \mathrm{Nm}$.

\section{Remarks}

It was not the goal of this research to address the possible use of a quantifiable method outside a gait laboratory, which if successful, could allow wide implementation in the prosthetic industry. However, the moments were measured with the iPecs to test our own inverse dynamics method. The results show some similarities, as well as some discrepancies between the portable method (iPecs-system) and the gait laboratory (GRAIL-system). Both reported equal significance before and after changes, indicating both methods to be valid in measuring the ESRM data. However, the consistent measurement error observed, with a valgus-orientated ESRM by the iPecs system and a neutral in the inverse dynamics methods, suggests a non-optimal defined inverse dynamics method, which should be addressed in a future alignment study. 